\title{
ARIAS: Automated Retinal Image Analysis System
}

Nashwa El-Bendary, Aboul Ella Hassanien, Emilio Corchado, and Robert C. Berwick

\begin{abstract}
In this paper, a system for automated analysis of retinal images is proposed. This system segments blood vessels in retinal images and recognizes the main features of the fundus on digital color images. The recognized features were defined as blood vessels, optic disc, and fovea. An algorithm called 2D matched filters response has been proposed for the detection of blood vessels. Also, automatic recognition and localization methods for optic disc and fovea have been introduced and discussed. Moreover, a method for detecting left and right retinal fundus images has been presented.
\end{abstract}

\section{Introduction}

Medical imaging has become a major tool among the modern health care community because of the visual documentation and record storing for the patients and for its ability to extract information about many diseases [1]. Discovering visual information can lead to evidences for the diagnosis of a disease; typical examples could be the X-rays or Magnetic Resonance Imaging (MRI). Manual inspection and labeling of a large image data set by medical experts is a tedious and slow task that inevitably leads to a decreasing performance over time for the same expert. This is

Nashwa El-Bendary

Arab Academy for Science,Technology, and Maritime Transport, Cairo, Egypt e-mail: nashwa_m@aast.edu

Aboul Ella Hassanien

Cairo University, Faculty of Computers and Information, Cairo, Egypt e-mail: aboitegypt@gmail.com

Emilio Corchado

University of Salamanca, Faculty of Science, Spain e-mail: escorchado@usal.es

Robert C. Berwick

Massachusetts Institute of Technology (MIT), USA e-mail: berwick@csail.mit.edu 
due to the fact that two experts could get different conclusions when they are given the same visual data because of the difficulty related to the data analyzed or the varying image conditions. Therefore, using large databases for medical imaging brings specific challenges, such as the necessity of tools that can tackle and process this large amount of data in useful and helpful ways for medical experts. Also, image data analysis may require the collaboration between experts, possibly in different locations. This is true in a telemedicine environment, where timely responses are a critical requirement [1]. These most desirable features for a computerized medical image analysis system are (i) Facilitating the automatic/semi-automatic image analysis by establishing a common repeatable procedure and accordingly increasing the performance and reliability of the entire analysis. (ii) Supporting image storage and management, allowing the collaboration between experts in different locations for the different studies.

In ophthalmology, the retinal analysis is a key field for the early diagnosis of several diseases. It is clear that in this environment, it is necessary for a medical imaging computer system to fulfill the features previously described is necessary. Some telemedicine studies have been performed in the field of ophthalmology [2]. The automated retinal image analysis system (ARIAS) proposed in this paper provides the ophthalmologists with a framework where retinal images can be managed, analyzed and stored in order to process retinal images to assess the condition of the patients.

The rest of this paper is organized as follows. Section (2) gives a brief overview of retina and retinal images. Section (3) presents the architecture of the proposed automated retinal image analysis system (ARIAS) and describes its phases. Classifiers and results are discussed in section (4). Finally, Section (5) summarizes conclusions and discusses future work.

\section{An Overview}

The eye is an organ associated with vision. It is housed in a socket of bone called the orbit and is protected from the external air by the eye lids [3]. The retina of an eye is an essential part of the central visual pathways that enable humans to visualize the real world. Retinal images tell us about retinal, ophthalmic, and even systematic diseases [4]. The retina is a multi-layered sensory tissue that lines the back of the eye.It contains millions of photo receptors that capture light rays and convert them into electrical impulses [5]. These impulses travel along the optic nerve to the brain where they are turned into images.In a normal fundus images, the Optic Disc (OD) is brighter than any part of the retina and is normally circular in shape [3]. It is also the entry and exit point for nerves entering and leaving the retina to and from the brain. A typical retina fundus image, the bright OD. Retinal images are being used by ophthalmologists to aid in diagnoses, to make measurements, and to look for 
change in lesions or severity of diseases. Particularly, the appearance of blood vessel is an important indicator for many diagnoses, including diabetes, hypertension, and arteriosclerosis. An accurate detection of blood vessels provides us the basics for the measurement of a variety of features that can then be applied to tasks like diagnosis, treatment evaluation, and clinical study. In addition, the detected vessel network supports the localization of the optic nerve and the multimodal/temporal registration of ocular fundus images $[4,6]$.

\section{Automated Retinal Image Analysis System (ARIAS)}

The proposed automated retinal image analysis system in this paper is composed of four main phases; pre-processing, segmentation, registration, and identification phases. Figure 1 describes the structure of the proposed system.

\subsection{Pre-processing Phase}

The main objective of preprocessing techniques is to attenuate image variation by normalizing the original retinal image against a reference model or data set for subsequent viewing, processing or analysis. Variations typically arise within the same image (intra-image variability) as well as between images (inter-image variability) and in order to obtain meaningful information from an image, it is necessary to compensate for this variability. Intra-image variations arise due to differences in light diffusion, the presence of abnormalities, variation in fundus reflectivity and fundus thickness. Inter-image variability is particularly important for longitudinal studies. Differences between images may be caused by factors including differences in cameras, illumination, acquisition angle and retinal pigmentation [7]. The preprocessing of both monochromatic and color retinal images may be loosely classified in terms of the correction for non-uniform illumination, contrast enhancement and color normalization. Before we enter the dominant processing stages, we manipulate a retinal image in such way that it later performs the basic standards for the following processing. The standards that should be fulfilled are the dimensions of the image, color and the illumination standard. To overcome the problem of a poor intensity image we normalize we normalize the image to can re-scaling or offsetting the data as necessary. To achieve this we abstract the lowest intensity value from every pixel in the image and secondly we divide all pixel intensities by the highest one. 


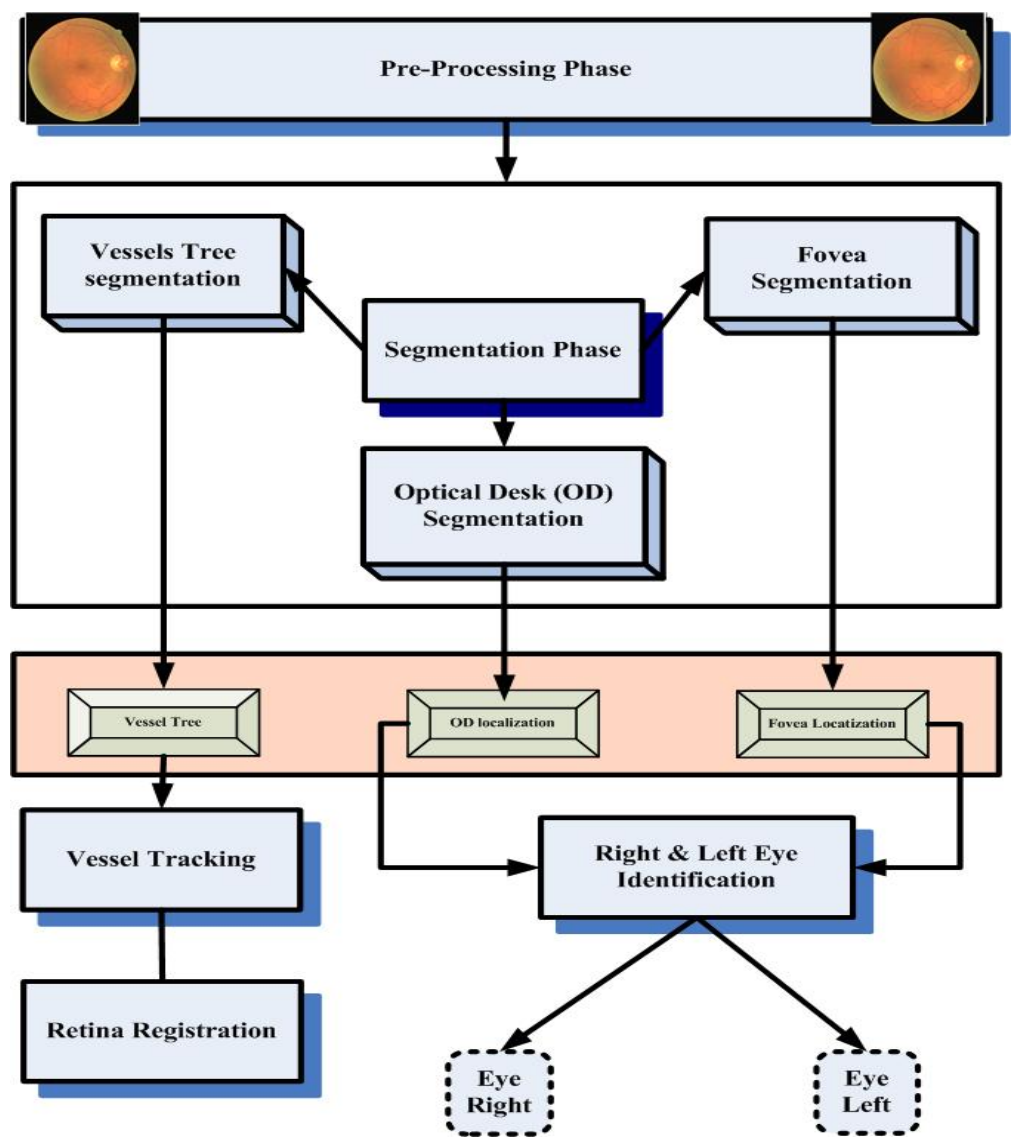

Fig. 1 Baseline structure of the retinal image analysis system

\subsection{Segmentation Phase}

Segmentation involves dividing images into subsections that are of particular interest, such as defining areas of an image that are appropriate to be subsequently analyzed, or finding circles, lines or other shapes of interest. Segmentation can stop when such objects of interest have been isolated. Retinal vessel segmentation is an essential step of the diagnoses of various eye diseases [8]. Automating the segmentation process provides several benefits including minimizing subjectivity and eliminating a painstaking, tedious task. Segmentation of retinal vasculature from the retinal images is used in many medicine disciplines, e.g. eye disease identification, biometrics or image registration. 


\subsubsection{Fovea Segmentation}

The fovea is a small, slightly concave area without retinal capillaries and its detection is very important for ocular diagnosis systems [6]. It appears as a dark area, in contrast to the rest of the retina where retinal vessels are enhanced by the fluorescent liquid as in figure 2. The fovea is so important because some specialized cells that provide central vision lie in it. Therefore, the severity of a lesion partially depends on its distance to the fovea [9]. Fovea segmentation process is described in algorithm (1).

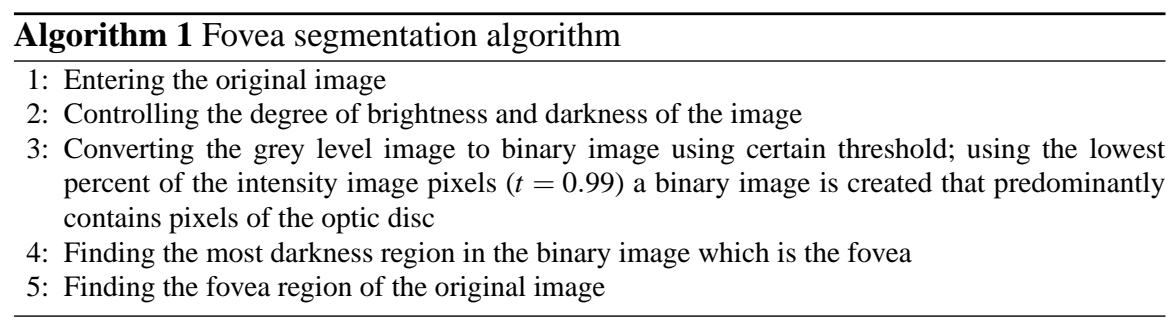

\subsubsection{Optic Disc (OD) Segmentation}

Localization of the optic disc is the identification of the center of the disc either by specifying the center of the optic disc or placing a mask within a particular region of the retina [8]. Segmentation of the optic disc usually refers to the subsequent task of determining the contour of the disc. Optic Disc (OD) localization provides a great medical importance in helping other retina image analysis tasks such as vessels tracking, fovea localization, recognition of left and right eyes and finally image registration [8]. The disc center and contour are often prerequisite landmarks in establishing a frame of reference for identifying retinal anatomy and pathology. The dimensions of the disc may be used to calibrate measurements in retinal images; for example, the fovea, which is the center of vision, is located between the major temporal retinal vessels approximately 2.5 disc diameters temporal to the temporal edge of the optic disc [7]. Localizing the optic disc is also a prerequisite for the computation of some important diagnostic indices for hypertensive sclerotic retinopathy based on vasculature, such as central retinal artery equivalent and central retinal vein equivalent [8]. Finally, the retinal vessels emanate from the center of the optic disc. Therefore, the disc may be used as a starting point for vessel tracking methods. Algorithm (2) shows the steps of OD segmentation process. 


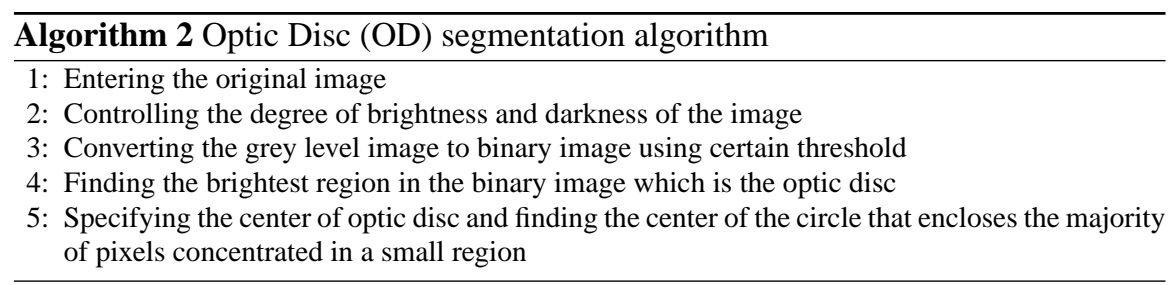

\subsubsection{Vessel Tree Segmentation}

The segmentation and measurement of the retinal vessels is of primary interest in the diagnosis and treatment of a number of systemic and ophthalmologic conditions. The accurate segmentation of the retinal blood vessels is often an essential prerequisite step in the identification of retinal anatomy and pathology. In addition, the segmentation of the vessels is useful for image registration or spatial alignment of images. The registration of images, which are often acquired using different modalities, is a critical operation in parametric imaging and the longitudinal monitoring of retinal appearance. In this paper, an algorithm called Matched Filter Response (MFR) has been proposed for the segmentation of blood vessels in a retinal image.

The strength of the MFR algorithm is coded in gray scale: the darker a pixel, the stronger the response. Notice that the strong responses may be not vessels, so it is necessary to use probing threshold for ensuring that strong responses are vessels [7, 10]. Algorithm (3) represents the steps for vessel tree segmentation.

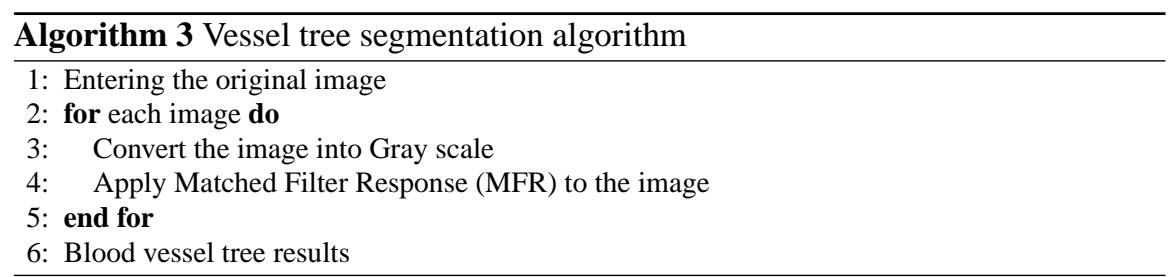

\subsection{Vessel Tree Tracking Phase}

The proposed algorithm for vessel tree tracking consists of four steps; (1) Generate the histogram of the green component and obtain new processed image, (2) Apply the matched filter to the histogram matched image to enhance blood vessels, (3) Use probing threshold to distinguish between vessel segments and the background in the MFR image, and (4) Label filtering technique is used to remove the misclassified pixels. Matched filtering for the detection of the vasculature convolves a 2D kernel with the retinal image. The kernel is designed to model some feature in the image 
at some unknown position and orientation, and the matched filter response (MFR) indicates the presence of the feature [7]. The concept of matched filter detection is used to detect piecewise linear segments of blood vessels in retinal images. Blood vessels usually have poor local contrast. The two dimensional matched filter kernel is designed to convolve with the original image in order to enhance the blood vessels [11]. A prototype matched filter kernel is expressed in equation (1).

$$
f(x, y)=-e^{\frac{-x^{2}}{2 \sigma^{2}}} \quad|y| \leq \frac{L}{2}
$$

where $L$ is the length of the segment for which the vessel is assumed to have a fixed orientation. Three primary characteristics determine properties of the kernel. Vessels, which usually have a limited curvature and may be approximated by piecewise linear segments; the diameter of the vessels that decreases as they move radially outward from the optic disc; and the cross-sectional pixel intensity profile of these line segments that approximates a Gaussian curve.

The segments created by simple threshold are used only to locate a set of starting points to initialize the probe queue. The segments themselves will not appear in the final segmentation unless the probing procedure causes their "reappearance" and classification as vessel. This process of initialization allows the pixels with a strong response to the matched filter to act as candidate vessels, with the design that not all need necessarily become part of the final segmentation. Each pixel in the probe queue is used as a starting point for threshold probing. The probing is iterative; the iterations are used to determine an appropriate threshold for the area being probed. The initial threshold is the MFR image value at the starting pixel. In each iteration, a region is grown from the start pixel, using a conditional paint-fill technique [8].

The paint-fill spreads across all connection pixels that are not already labeled and that are above the current threshold. Once the paint-fill is complete, the desired attributes of the grown region are tested. If the region passes the tests, then the threshold is decreased by one, and a new iteration begins [12].

\subsection{Identification Phase}

Automatic segmentation of blood vessels in retinal images is very important in early detection and diagnosis of many eye diseases. It is an important step in screening programs for early detection of diabetic retinopathy, registration of retinal images for treatment evaluation (i.e., to follow the evaluation of some lesions over time or to compare images obtained under different conditions), generating retinal maps for diagnosis and treatment of age-related two macular degeneration, or locating the optic disc and the fovea [13]. 


\section{Experimental Results and Discussion}

Optic disc contour segmentation is usually performed after identifying the approximate center of the disc. Identifying the contour of the optic disc is a non-trivial problem. The natural variation in the characteristics of the optic disc including the previously described differences in pigmentation and myelination of the nerve fiber layer are significant problems for defining the contour of the disc. Blood vessels may cross the boundary of the optic disc obscuring the rim of the disc with the edges of vessels also acting as significant distracters [7, 14].

The optic disc is usually the brightest component on the funds, and therefore a cluster of high intensity pixels and high variances will identify the optic location so control process of the brightness degree is done to obtain the optic disc. Figure 2 presents OD localization and segmented vessel tree.

The human retina is a thin tissue composed of neural cells that is located in the posterior portion of the eye. The retina has ten layers and it presents the surface inside the back of the eye, upon which images that have passed through the pupil are focused. The proposed system contains six analysis of retinal image. With the segmented optic disc in the candidate Region of Interest (RoI) area, we extract the vessel information from the optic disc via its green channel. It is found in our experiments that the central retinal artery and vein are most distinct in this channel. The optic disc is divided into left and right halves. The extracted vessels from the optic disc are then summed up in its columns and projected horizontally across the x-axis. The pixel intensities in each half are compared against each other. The half which indicates lower pixel intensity denotes that it is the temporal sector. If the temporal sector is on the right half, it signifies a right eye, and vice versa. If the fovea is in the right side of the optic disc, then the eye is on the right otherwise the retina is on the left eye, as shown in Figure 3.

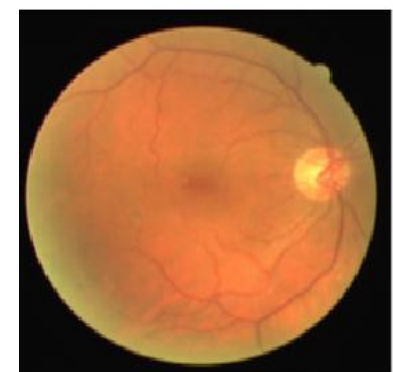

(a) Original image

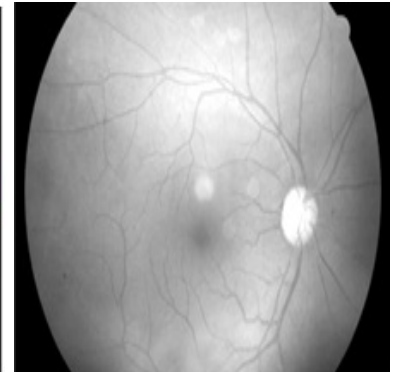

(b) The fovea location

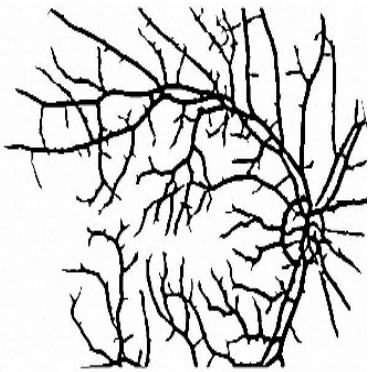

(c) Segmented blood vessel

Fig. 2 Fovea localization and Segmented vessel tree 


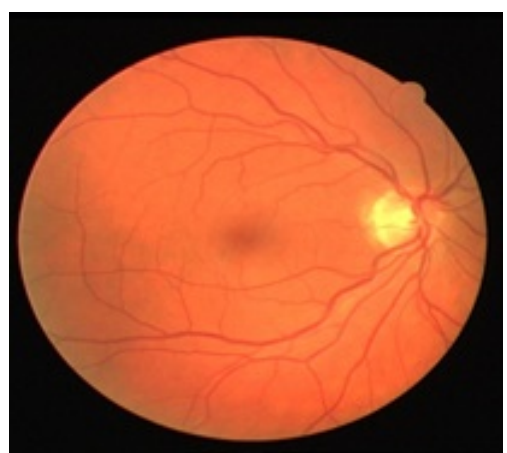

(a) Right eye

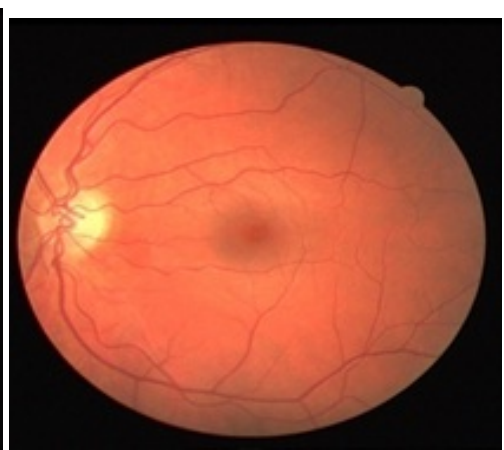

(b) Left eye

Fig.3 Distinguishing between right and left eyes

\section{Conclusions and Future Work}

In this paper, an automated system for retinal images analysis has been presented. The proposed system segments blood vessels in retinal images and recognizes the main features of the fundus on digital color images. The recognized features were defined as the blood vessels, optic disc, and fovea. An algorithm called 2D matched filters response has been proposed for the detection of blood vessels. Also, automatic recognition and location methods for optic disc and fovea have been introduced and discussed. Moreover, a method for detection of left and right retinal fundus images has been presented for deciding whether the infected eye is the left or the right eye.

Retinal image analysis can lead to early detection of several pathologies such as hypertension or diabetes. Screening process requires the evaluation of a huge amount of visual data and, usually, the collaboration between different experts and different health care centers. These usual routines demand new fast and automatic solutions to deal with these situations. Web-based systems for image analysis in the retinal imaging field are required to provide a fast and reliable work environment for retinal experts.

\section{References}

1. Ortega M., Barreira N., Novo J., Penedo MG., Pose-Reino A. and Gmez-Ulla F. (2010) Sirius: A web-based system for retinal image analysis. International Journal of Medical Informatics, 79(10):722-732.

2. Richter G.M., Williams S.L., Starren J., Flynn J.T., and Chiang M.F. (2009) Telemedicine for retinopathy of prematurity diagnosis: evaluation and challenges. Survey of Ophthalmology, 54(6):671-685.

3. Aibinu A.M., Iqbal M.I., Shafie A.A., Salami M.J.E., and Nilsson M., (2010) Vascular intersection detection in retina fundus images using a new hybrid approach. Elseiver Journal of 
Computers in Biology and Medicine, 40(1):81-89.

4. Xiaoyi J. and Daniel M. (2001) Blood Vessel Detection in Retinal Images by Shape-Based Multi-threshold Probing. Proceedings of the 23rd DAGM-Symposium on Pattern Recognition'2001, September 12-14, 38-44.

5. Aibinu A.M., Iqbal M.I., Nilsson M., and Salami M.J.E. (2007) A new method of correcting uneven illumination problem in fundus images. The International Conference on Robotics, Vision, Information, and Signal Processing,Penang, Malaysia,445449.

6. Lowell J., Hunter A., Steel D., Basu A., Ryder R., and Fletcher E. (2004) Optic nerve head segmentation. IEEE. Transactions on Medical Imaging, 23(2):256-264.

7. Winder R.J., Morrow P.J., McRitchie I.N., Bailie J.R., and Hart P.M. (2009) Algorithms for digital image processing in diabetic retinopathy," Computerized Medical Imaging and Graphics, 33(8):608-622.

8. Niall P., Tariq M. A., Thomas M.,Ian J. D., Baljean D., Robert H. E., Kanagasingam Y., and Ian J. C. (2006) Retinal image analysis: Concepts, applications and potential. Progress in Retinal and Eye Research, 25(1):99-127.

9. Sekhar S., Al-Nuaimu W. and Nandi A. K. (2008) Automated localisation of optic disc and fovea in retinal fundus images. Proceedings of the 16th European Signal Processing Conference (EUSIPCO-2008), Lausanne, Switzerland.

10. Wong Li Y., Rajendra Acharya U., Venkatesh Y.V., Caroline C., Lim Choo M. and Ng E.Y.K.(2008) Identification of different stages of diabetic retinopathy using retinal optic images. Information Sciences, 178(1):106-121.

11. Echevarria P., Miller T. and Meara J.O. (2004) Blood Vessel Segmentation in Retinal Images," Archives of Ophthalmology, 122(4):552563.

12. Hoover A., Kouznetsova V., and Goldbaum M. (2000) Locating blood vessels in retinal images by piece-wise threshold probing of a matched filter response. IEEE Trans. Medical Imaging, 19(3):203-210.

13. Salem S.A., Salem N.M. and Nandi A.K. (2006) Segmentation of retinal blood vessels using a novel clustering algorithm. Proceedings of The 14th European Signal Processing Conference - EUSIPICO' 2006, Florence, Italy, September 4-8, 2006.

14. Ryan N., Heneghan C., and Philip de C. (2004) Registration of digital retinal images using landmark correspondence by expectation maximization. Image and Vision Computing, 22(11):883-898. 